\title{
Simulation study of the plasma-brake effect
}

\author{
P. Janhunen ${ }^{1, *}$ \\ ${ }^{1}$ Finnish Meteorological Institute, POB-503, 00101, Helsinki, Finland \\ *also guest professor at Tartu University, Tartu, Estonia \\ Correspondence to: P. Janhunen (pekka.janhunen@fmi.fi)
}

Received: 23 January 2014 - Revised: 19 August 2014 - Accepted: 28 August 2014 - Published: 7 October 2014

\begin{abstract}
Plasma brake is a thin, negatively biased tether that has been proposed as an efficient concept for deorbiting satellites and debris objects from low Earth orbit. We simulate the interaction with the ionospheric plasma ram flow with the plasma-brake tether by a high-performance electrostatic particle in cell code to evaluate the thrust. The tether is assumed to be perpendicular to the flow. We perform runs for different tether voltage, magnetic-field orientation and plasma-ion mass. We show that a simple analytical thrust formula reproduces most of the simulation results well. The interaction with the tether and the plasma flow is laminar (i.e. smooth and not turbulent) when the magnetic field is perpendicular to the tether and the flow. If the magnetic field is parallel to the tether, the behaviour is unstable and thrust is reduced by a modest factor. The case in which the magnetic field is aligned with the flow can also be unstable, but does not result in notable thrust reduction. We also correct an error in an earlier reference. According to the simulations, the predicted thrust of the plasma brake is large enough to make the method promising for low-Earth-orbit (LEO) satellite deorbiting. As a numerical example, we estimate that a $5 \mathrm{~km}$ long plasma-brake tether weighing $0.055 \mathrm{~kg}$ could produce $0.43 \mathrm{mN}$ breaking force, which is enough to reduce the orbital altitude of a $260 \mathrm{~kg}$ object mass by $100 \mathrm{~km}$ over 1 year.
\end{abstract}

Keywords. Ionosphere (active experiments)

\section{Introduction}

Plasma brake (Janhunen, 2009, 2010) is an efficient, propellantless concept for deorbiting low-Earth-orbit (LEO) satellites. The plasma brake is a very thin negatively charged tether which, when charged, causes a braking force by creating enhanced Coulomb drag with ambient ionospheric plasma ram flow. The plasma-brake tether is somewhat similar to the better-known electrodynamic tether (Sanmartin et al., 1993), but is much thinner and uses electrostatic, rather than magnetic, forces.

Because the plasma-brake four-wire ultrasonically bonded tether is thin, it is lightweight, $11 \mathrm{~g} \mathrm{~km}^{-1}$ (Seppänen et al., 2013) and does not form an appreciable threat to other satellites, even in the case of tether breakage. A broken piece of plasma-brake tether deorbits itself quickly because of electromagnetic forces and neutral drag, and, even if the tether piece were to collide with a satellite, the $25-50 \mu \mathrm{m}$ wires draw only $\sim 0.1 \mathrm{~mm}$ deep scratches on its surface. Said electromagnetic forces are a passive Coulomb drag effect and, to a lesser extent, the passive electrodynamic tether effect. The broken tether piece experiences an orbital motion-induced natural electric field, and whenever this field has a component along the tether, one end of the tether gets charged negatively and the other one positively, with respect to the local plasma, causing electrostatic Coulomb drag and electromagnetic Lorentz drag. Although the passive electromagnetic drag effects are weaker than the active ones, the very low specific mass of the tether $\left(\sim 0.01 \mathrm{~kg} \mathrm{~km}^{-1}\right)$ typically makes passive deorbiting of a broken tether piece fast.

Maintaining a tether at positive voltage in the solar wind requires an electron gun (Janhunen et al., 2010, 2013) that pumps out negatively charged particles from the system and thereby cancels the tether's gathered plasma current. Likewise, maintaining a tether at negative voltage can be accomplished with a positive ion gun. However, in LEO, the maintenance of the plasma-brake tether's negative voltage does not require, in most cases, an ion gun, since the satellite's conducting body can be used as the current balancing electron gathering surface (Janhunen, 2010). If the satellite's grounded and conducting surface area is insufficiently small for this purpose, a relatively short positively biased tether made of similar material to the main tether could be used for gathering the required balancing electron current. 
The topic of the paper is to use a realistic electrostatic particle-in-cell (PIC) simulation to model the interaction between the negatively biased plasma-brake tether and the surrounding ionospheric plasma ram flow, which moves at a typical satellite orbital speed of $7.5 \mathrm{~km} \mathrm{~s}^{-1}$. We run the code with different voltages, different magnetic-field orientations and different ion species, and we determine the thrust per tether length in each case. We will also present a simple analytical formula for plasma-brake thrust, which reproduces the simulation results.

\section{Simulation code}

We use a two-dimensional explicit electrostatic kinetic PIC simulation model (Birdsall and Langdon, 1991). The functionality of the code is similar to that which we used earlier to model the positively biased E-sail tether (Janhunen and Sandroos, 2007), but the code is vectorised with AgnerVector library (Fog, 2013) and parallelised with the standard Message Passing Interface (MPI) and OpenMP tools. We run the code with real electron and ion masses.

We simulate a negatively charged tether in plasma flow, which is caused by the satellite's orbital motion through the ionosphere. We use a Cartesian coordinate system, where $X$ is antiparallel with the tether-perpendicular component of the flow, $Z$ is parallel to the tether and $Y$ completes a right-handed coordinate system. For convenience, we take the plasma flow to be perpendicular to the tether, which implies no loss in generality. The simulation is two-dimensional in the tether-perpendicular plane. A constant external magnetic field is employed in some runs. By default, the plasma is cold oxygen plasma (temperature $0.1 \mathrm{eV}$ ) and it flows at $7.5 \mathrm{~km} \mathrm{~s}^{-1}$, which corresponds to $4.7 \mathrm{eV}$ bulk-flow ion energy. The tether bias voltage is typically a few hundred volts negative. The parameters of all reported runs are given in Table 1 . The simulations are initialised from vacuum and the plasma flow starts entering the box at $t=0$.

Above $\sim 500 \mathrm{~km}$, where the plasma brake is relevant (at lower altitude, the neutral drag typically brings satellites down rapidly enough), ionospheric plasma consists mainly of $\mathrm{O}^{+}(16 \mathrm{amu}), \mathrm{N}^{+}(14 \mathrm{amu}), \mathrm{He}^{+}(4 \mathrm{amu})$ and $\mathrm{H}^{+}(1 \mathrm{amu})$ ions. The main species are $\mathrm{O}^{+}$and $\mathrm{H}^{+}$; the minor species $\mathrm{N}^{+}$can be summed to $\mathrm{O}^{+}$, because the masses are approximately similar. The abundance of $\mathrm{O}^{+}$decreases with altitude. At all altitudes, solar activity tends to increase the oxygen abundance. For the plasma brake, the most relevant environment is $\mathrm{O}^{+}$plasma, because proton plasma produces order of magnitude smaller thrust, and helium is usually not a dominant species. For this reason, the main emphasis in this paper is on $\mathrm{O}^{+}$plasma.

Runs reported in this paper used 16 nodes on a Cray XC30. The XC30 consists of compute nodes. Each node has 2 processor chips and each chip has $102.6 \mathrm{GHz}$ execution cores. The runs reported here used $16 \mathrm{XC} 30$ nodes and
Table 1. Simulation parameters of the V400 run (hereafter referred to as "run Baseline").

\begin{tabular}{lll}
\hline Parameter & Symbol & Value \\
\hline Grid size & & $512 \times 512$ \\
Grid spacing & $\Delta x$ & $7.3 \mathrm{~mm}$ \\
Normalised spacing & $\Delta x / \lambda \mathrm{De}$ & 0.54 \\
$X$ grid domain & & $-2.34 \ldots 1.4 \mathrm{~m}$ \\
$Y$ grid domain & & $-1.87 \ldots 1.87 \mathrm{~m}$ \\
Timestep & $\Delta t$ & $5.84 \mathrm{~ns}$ \\
Normalised time step & $\omega_{\mathrm{pe}} \Delta t$ & 0.057 \\
Run duration & $t_{\mathrm{max}}$ & $2.43 \mathrm{~ms}$ \\
Number of time steps & & 417000 \\
Electrons per cell & $N_{0}$ & $500(\mathrm{in} \mathrm{plasma} \mathrm{stream})$ \\
Number of particles & & $\sim 52.4 \mathrm{M}$ \\
Plasma density & $n_{\mathrm{O}}$ & $3 \times 10^{10} \mathrm{~m}^{-3}$ \\
Ion mass & $m_{\mathrm{i}}$ & $16 \mathrm{amu}\left(\mathrm{O}^{+}\right)$ \\
Plasma drift & $v_{\mathrm{o}}$ & $7.5 \mathrm{~km} \mathrm{~s} \mathrm{~s}^{-1}$ \\
Electron temp. & $T_{\mathrm{e}}$ & $0.1 \mathrm{eV}$ \\
Ion temp. & $T_{\mathrm{i}}$ & $0.1 \mathrm{eV}$ \\
Magnetic field & $B_{x}, B y, B_{z}$ & $0 \mathrm{nT}$ \\
Tether voltage & $V_{\mathrm{w}}$ & $337.436 \mathrm{~V}$ \\
Tether electric radius & $r_{\mathrm{w}}^{*}$ & $1 \mathrm{~mm}$ \\
\hline
\end{tabular}

achieved $4.4 \times 10^{9}$ particle propagations per second, which corresponds to $\sim 1$ Tflops single precision floating point performance.

\section{Results}

\subsection{Oxygen plasma without magnetic field}

We first run the code with oxygen plasma and without magnetic field for different values of the tether potential $V_{\mathrm{w}}$. The runs are detailed in Table 2. The vacuum potential of the tether (the voltage difference between the tether and the simulation box boundary if there is no plasma inside the box) is an input parameter to the simulation. After the simulation, the true potential of the tether is calculated by evaluating the plasma potential at the tether and adding it to the vacuum potential. For this reason, the tether potential values $V_{\mathrm{w}}$ are not round numbers. We label the runs partly by their vacuumpotential values.

Figure 1 shows the simulated thrust (determined from particle momentum balance averaged over the last third of the run) with open circles. Coloured marks in Fig. 1 are results from some runs containing a magnetic field and they are described in later subsections below. The solid line is the following analytical formula, inspired by our earlier work (Janhunen, 2009, Eq. 20):

$$
\frac{\mathrm{d} F}{\mathrm{~d} z}=3.864 \times P_{\mathrm{dyn}} \sqrt{\frac{\epsilon_{\mathrm{o}} \tilde{V}}{e n_{\mathrm{o}}}} \exp \left(-V_{i} / \tilde{V}\right),
$$


Table 2. List of performed runs. Only differences to run Baseline are mentioned. Runs ending with "g” have larger $768 \times 768$ grid. The Long runs have $23.4 \mathrm{~ms}$ duration. The "Rel. thrust" column tells how much the thrust differs from Eq. (1) prediction.

\begin{tabular}{llrrrl}
\hline Run & Parameters & Thrust $/ \mathrm{nNm}^{-1}$ & Eq. $(1) / \mathrm{nNm}^{-1}$ & Rel. thrust & Nature \\
\hline V50 & $V_{\mathrm{w}}=41.0 \mathrm{~V}$ & 7.06 & 10.6 & $-33 \%$ & Stable \\
V100 & $V_{\mathrm{w}}=82.4 \mathrm{~V}$ & 19.4 & 19.8 & $-2 \%$ & Stable \\
V150 & $V_{\mathrm{w}}=125 \mathrm{~V}$ & 27.3 & 26.7 & $+2 \%$ & Stable \\
V200 & $V_{\mathrm{w}}=168 \mathrm{~V}$ & 32.8 & 32.3 & $+2 \%$ & Stable \\
V300 & $V_{\mathrm{w}}=252 \mathrm{~V}$ & 40.5 & 41.2 & $-2 \%$ & Steady oscillation \\
V400 & Table $1, V_{\mathrm{w}}=337 \mathrm{~V}$ & 47.9 & 48.5 & $-1 \%$ & Steady oscillation \\
V500 & $V_{\mathrm{w}}=424 \mathrm{~V}$ & 54.5 & 54.9 & $-1 \%$ & Steady oscillation \\
V500g & $V_{\mathrm{w}}=424 \mathrm{~V}$ & 54.9 & 54.9 & $0 \%$ & Steady oscillation \\
V600g & $V_{\mathrm{w}}=512 \mathrm{~V}$ & 60.9 & 60.6 & $0 \%$ & Steady oscillation \\
V800g & $V_{\mathrm{w}}=689 \mathrm{~V}$ & 71.4 & 70.7 & $+1 \%$ & Steady oscillation \\
V1000g & $V_{\mathrm{w}}=869 \mathrm{~V}$ & 80.1 & 79.5 & $+1 \%$ & Steady oscillation \\
Bx & $B_{x}=30 \mu \mathrm{T}, V_{\mathrm{w}}=333 \mathrm{~V}$ & 51.1 & 48.2 & $+6 \%$ & Unstable \\
BxLong & $B_{x}=30 \mu \mathrm{T}, V_{\mathrm{w}}=311 \mathrm{~V}$ & 40.9 & 46.4 & $-12 \%$ & Unstable \\
By & $B_{y}=30 \mu \mathrm{T}, V_{\mathrm{w}}=338 \mathrm{~V}$ & 48.5 & 48.6 & $0 \%$ & Dying oscillation \\
Bz & $B_{z}=30 \mu \mathrm{T}, V_{\mathrm{w}}=335 \mathrm{~V}$ & 47.2 & 48.4 & $-2 \%$ & Unstable \\
BzLong & $B_{z}=30 \mu \mathrm{T}, V_{\mathrm{w}}=317 \mathrm{~V}$ & 38.7 & 46.9 & $-17 \%$ & Unstable \\
Helium & $m_{\mathrm{i}}=4 \mathrm{amu}, V_{\mathrm{w}}=350 \mathrm{~V}$ & 14.6 & 13.2 & $+11 \%$ & Unstable \\
Proton & $m_{\mathrm{i}}=1 \mathrm{amu}, V_{\mathrm{w}}=323 \mathrm{~V}$ & 5.06 & 3.24 & $+56 \%$ & Unstable \\
ProtonBzLong & $m_{\mathrm{i}}=1 \mathrm{amu}, V_{\mathrm{w}}=256 \mathrm{~V}$ & 2.91 & 2.91 & $0 \%$ & Unstable \\
V1000By & $B_{y}=30 \mu \mathrm{T}, V=870 \mathrm{~V}$ & 80.7 & 79.5 & $+2 \%$ & Steady oscillation \\
V1000BzLong & $B_{z}=30 \mu \mathrm{T}, V_{\mathrm{w}}=757 \mathrm{~V}$ & 53.8 & 74.1 & $-27 \%$ & Unstable \\
V1000Bx & $B_{x}=30 \mu \mathrm{T}, V_{\mathrm{w}}=856 \mathrm{~V}$ & 83.6 & 78.0 & $+7 \%$ & Stable \\
Te & $T_{\mathrm{e}}=0.3 \mathrm{eV}, V_{\mathrm{w}}=337 \mathrm{~V}$ & 48.7 & 48.5 & $0 \%$ & Dying oscillation \\
\hline
\end{tabular}

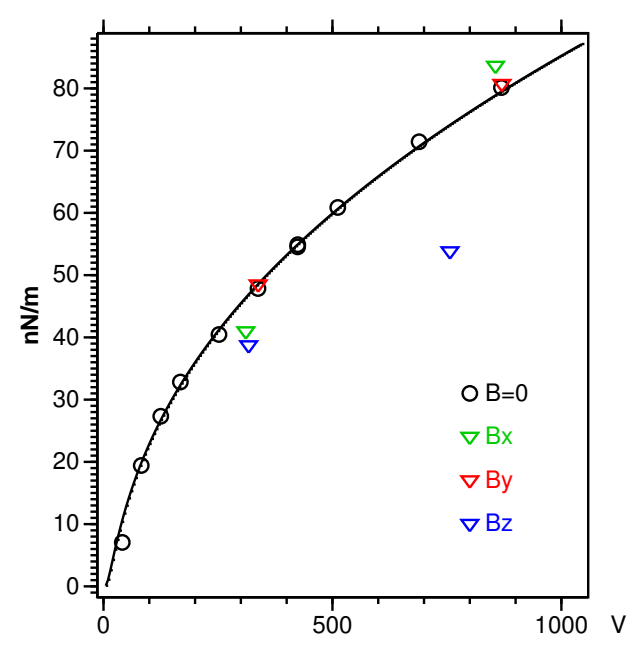

Figure 1. Different marks: thrust as function of tether voltage $V_{\mathrm{W}}$ in oxygen plasma with and without magnetic field, see legend in plot and Table 2 for run parameters. Solid line: Eq. (1).

where $P_{\mathrm{dyn}}=m_{\mathrm{i}} n_{\mathrm{o}} v_{\mathrm{o}}^{2}$ is the dynamic pressure, $m_{\mathrm{i}}$ is the ion mass $\left(m_{\mathrm{i}}=16 \mathrm{amu}\right.$ for oxygen plasma here), $v_{\mathrm{o}}$ is the plasma-flow speed relative to spacecraft (assumed to be perpendicular to the tether or else $v_{\mathrm{o}}$ denotes only the perpendicular component):

$$
\tilde{V}=\frac{V_{\mathrm{w}}}{\ln \left(\lambda_{\mathrm{D}}^{\mathrm{eff}} / r_{\mathrm{w}}^{*}\right)},
$$

where $r_{\mathrm{w}}^{*}$ is the tether's effective electric radius (Janhunen and Sandroos, 2007, Appendix A), $\lambda_{\mathrm{D}}^{\text {eff }}=\sqrt{\epsilon_{\mathrm{o}} V_{\mathrm{w}} /\left(e n_{\mathrm{o}}\right)}$ is the effective Debye length and $V_{\mathrm{i}}=(1 / 2) m_{\mathrm{i}} v_{\mathrm{o}}^{2} / e$ is the bulkion-flow energy in voltage units. The effective electric radius is approximately given by $r_{\mathrm{w}}^{*}=\sqrt{b r_{\mathrm{w}}}$, where $r_{\mathrm{w}}$ is the tether wire radius, typically $12.5-25 \mu \mathrm{m}$, and $b$ is the tether width, typically $2 \mathrm{~cm}$ (a rough value of $b$ is sufficient to know because $r_{\mathrm{w}}^{*}$ only enters into Eq. (1) logarithmically). In this paper, we use the value $r_{\mathrm{w}}^{*}=1 \mathrm{~mm}$. The numerical coefficient (3.864) in front of the expression has been selected to give a good fit to the present simulation results. This value is about 2.25 times larger than the value 1.72 used in our previous work (Janhunen, 2009). We think that this difference may arise because, in the earlier work, we used typical solarwind parameters so that the ratio $V_{\mathrm{w}} / V_{\mathrm{i}}$ was about 10 , while this ratio is about 100 in the present ionospheric case, so that the works explored different regions of the parameter space. In the ionospheric case, many ions are deflected backwards by the potential well around the tether, which increases the thrust markedly in comparison to the solar-wind case, where the much larger bulk-flow speed causes the ions to deflect more modestly. 


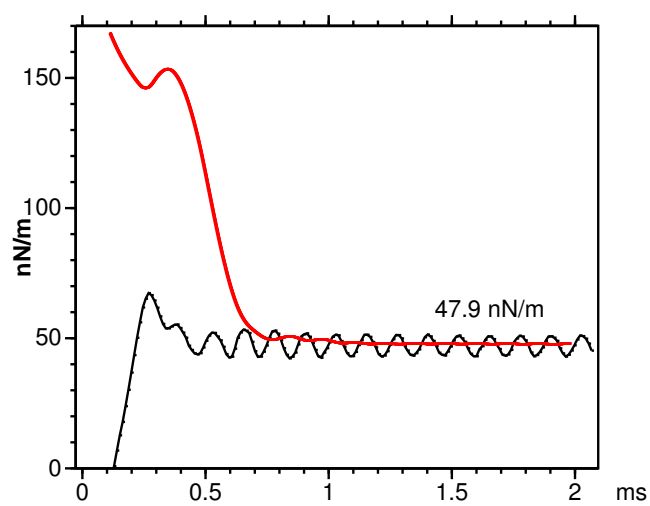

Figure 2. Time development of thrust in the Baseline, tether Coulomb force computation (black curve) and momentum-balance computation (red curve).

Figure 2 shows the time history of thrust during run V400 (hereafter referred to as "run Baseline"), computed by two complementary methods: the direct Coulomb force, acting on the tether (by evaluating the numerical gradient of the plasma potential at the tether position) and the total momentum $X$ component, lost by particles in between entering and leaving the simulation box. Figure 3 shows the twodimensional instantaneous electron and ion density at the final state of run Baseline.

Increasing the tether voltage makes the ion sheath larger. The highest voltage runs (marked with "g" in Table 2) were performed with an extended box size of $768 \times 768$ to accommodate the larger sheath. As a consistency check, run V500 was performed with both grid sizes. The difference in the determined thrust was minimal (Table 2, compare V500 and V500g).

The quasi-monochromatic oscillation, seen in Fig. 2, occurs in runs V300 and higher. In lower voltage runs, the oscillation is absent. When present, the oscillation neither increases nor decreases with time.

\subsection{Effect of magnetic field}

We performed three more runs with the same parameters as Baseline, but with each of the magnetic-field components in turn set to $30000 \mathrm{nT}$, a typical LEO field strength. The results are shown in Figs. 4-6. It is seen that, in runs $\mathrm{Bx}$ and $\mathrm{Bz}$, the oscillation, which was already present in run Baseline, is now increasing in amplitude. In contrast, in the By run, the oscillation is damped and is going to disappear. Thus, $B_{y}$ (magnetic field perpendicular to tether and flow) is stabilising, while flow-directed or tether-directed field is destabilising at the baseline voltage.

The final state of run Bx is shown in Fig. 7. The sheath surrounding the tether is unstable and radiates plasma waves in all directions in the $X Y$ plane. In the tether-fixed coordinate frame, the waves move approximately at the same phase
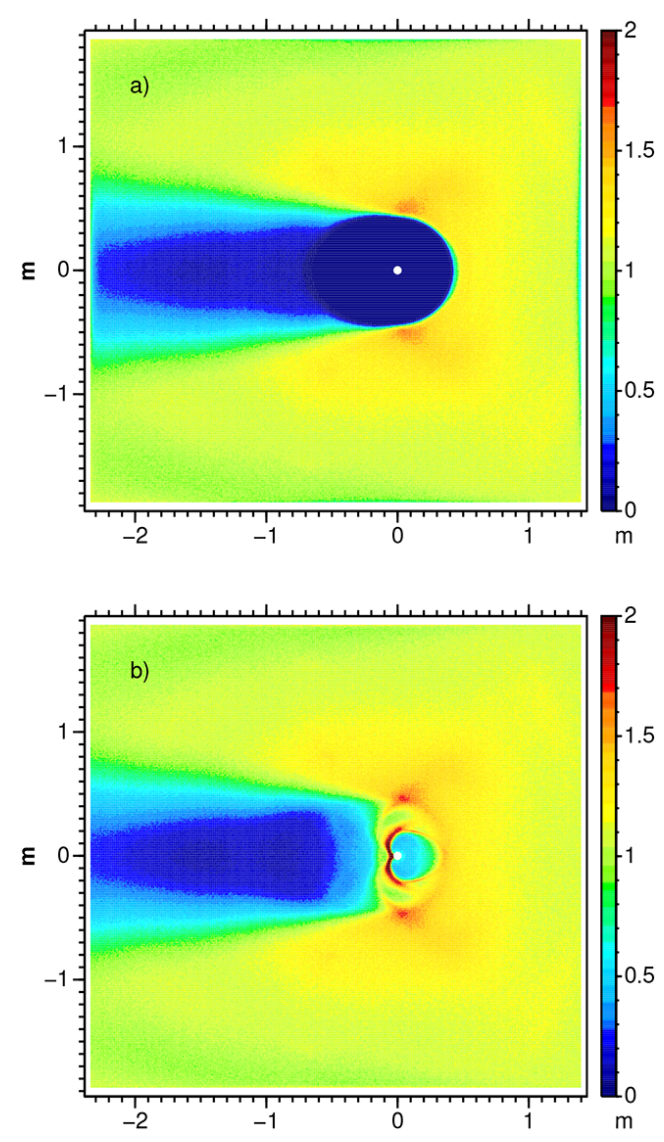

Figure 3. Electron density (a) and ion density (b) normalised to plasma-stream density in the final state $(t=2.43 \mathrm{~ms})$ of run Baseline.

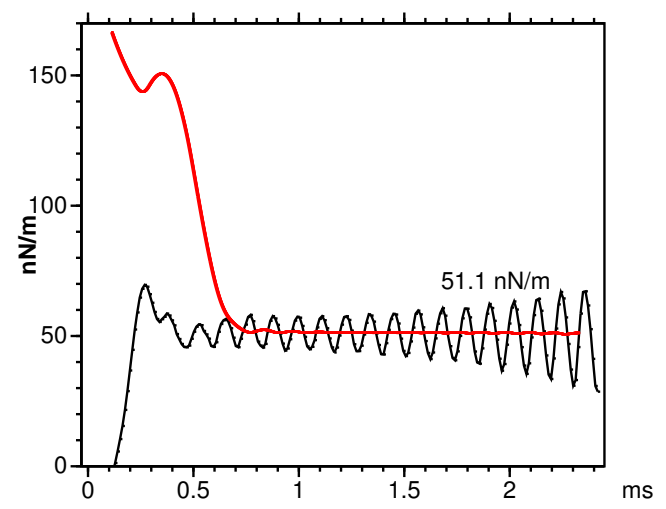

Figure 4. Run Bx: same as Fig. 2, but with $B_{x}=30000 \mathrm{nT}$.

speed as the bulk flow $\left(7.5 \mathrm{~km} \mathrm{~s}^{-1}\right)$. The waves appear to move with the ion flow, which is reflected by the tether's potential well. The incoming flow has a Mach number of $\approx 5$, with respect to ion-acoustic wave speed. Because of energy conservation, reflected ions move radially outwards with the same speed as the incoming flow. The tether's potential well fluctuates, which modulates the flow of outward-reflected 


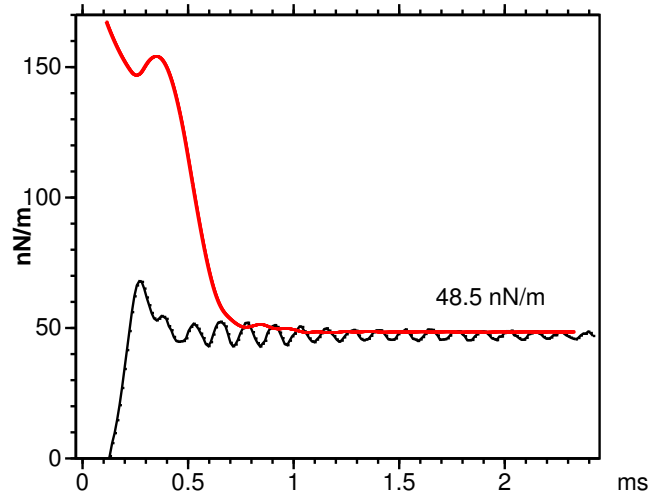

Figure 5. Run By: same as Fig. 2, but with $B_{y}=30000 \mathrm{nT}$.

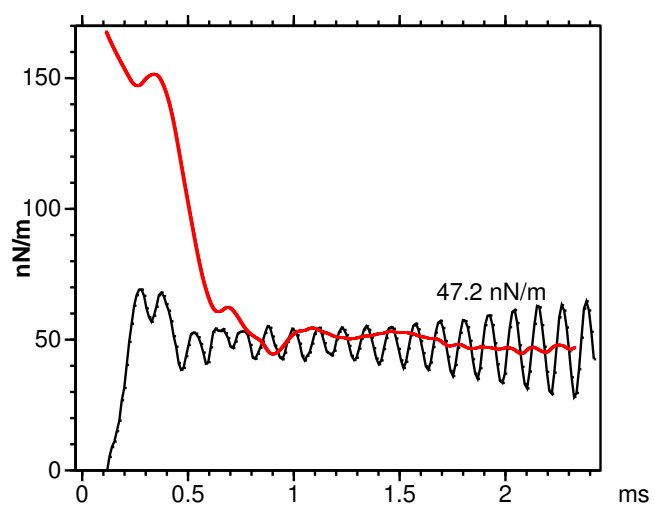

Figure 6. Run Bz: same as Fig. 2, but with $B_{z}=30000 \mathrm{nT}$.

ions. The modulations propagate outwards approximately at the same speed as the ions, because the ion-acoustic speed is less than the bulk velocity of the outward-moving ion population. In Fig. 7, one can also see that the $X$-directed magnetic field tends to restrict electron motion in $Y$, which creates some of the horizontal stripes seen in the figure (reddish stripes on positive $X$ ), visible in both electron and ion density, and emanating from the edges of the electron cavity.

Figure 8 shows the final state of run Bz. Again, the state is unstable and the tether's potential well radiates plasma waves. Additionally, the boundaries of the plasma wake behind the tether display some turbulent behaviour. This probably occurs because the magnetic field is now along the tether so that electrons respond to $X$ - and $Y$-directed electric fields by $\boldsymbol{E} \times \boldsymbol{B}$ drifting (similar to Kelvin-Helmholtz instability in plane perpendicular to $\boldsymbol{B}$ ). Not surprisingly, the horizontal stripes, which were visible in the Bx run (Fig. 7), are now absent.

\subsection{Effect of ion mass}

Figures 9 and 10 show thrust histories for helium and proton plasma, respectively, and with other parameters in their Baseline values. In both light-ion cases, an unstable oscillation is
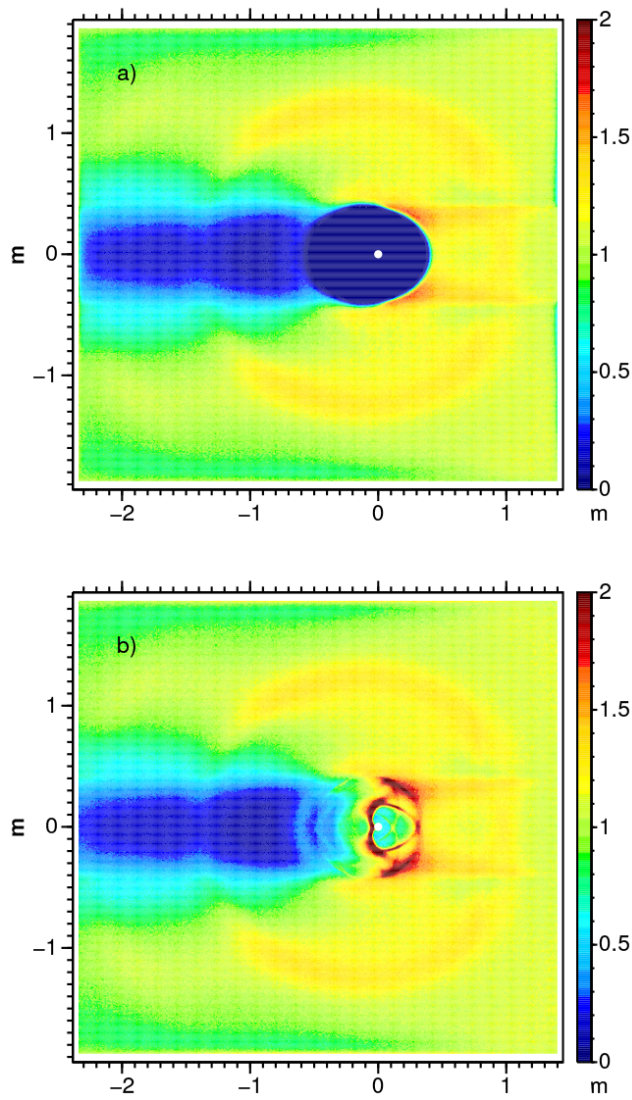

Figure 7. Electron density (a) and ion density (b) normalised to plasma-stream density in the final state $(t=2.43 \mathrm{~ms})$ of run $\mathrm{Bx}$.

present. The instability grows faster in the proton run than in the helium run, and has time to evolve into an intermittent, non-linear regime.

Figure 11 shows the final state of run Proton. Some ions have become trapped by the potential well, which has decreased the thrust to some extent (Fig. 10). The sheath emits plasma waves in all $X Y$ directions. The flow Mach number with respect to ion-acoustic speed is low (1.2), which is likely the reason why the wake behind the tether is rather short (the wake gets filled quickly from the boundaries because the ionthermal speed is almost as large as the flow speed). In Fig. 11, the behaviour of the densities near the inflow boundary (right side) shows some $Y$-directed striping and does not look completely natural. It is possible that the finite box size affects the result to some extent in this case. In the proton case (Fig. 11), it is also noteworthy that the emitted ion waves have a short enough wavelength that the electron density does not follow the ion density; in other words, the emitted wavelength is not much larger than the electron Debye length.

Formula 1 predicts $12.2 \mathrm{nN} \mathrm{m}^{-1}$ in the helium case and $2.92 \mathrm{nN} \mathrm{m}^{-1}$ in the proton case. The simulated values at $2.43 \mathrm{~ms}$ are larger (14.6 and $5.05 \mathrm{nN} \mathrm{m}^{-1}$, respectively), even though, in the proton run, some ion trapping had already 

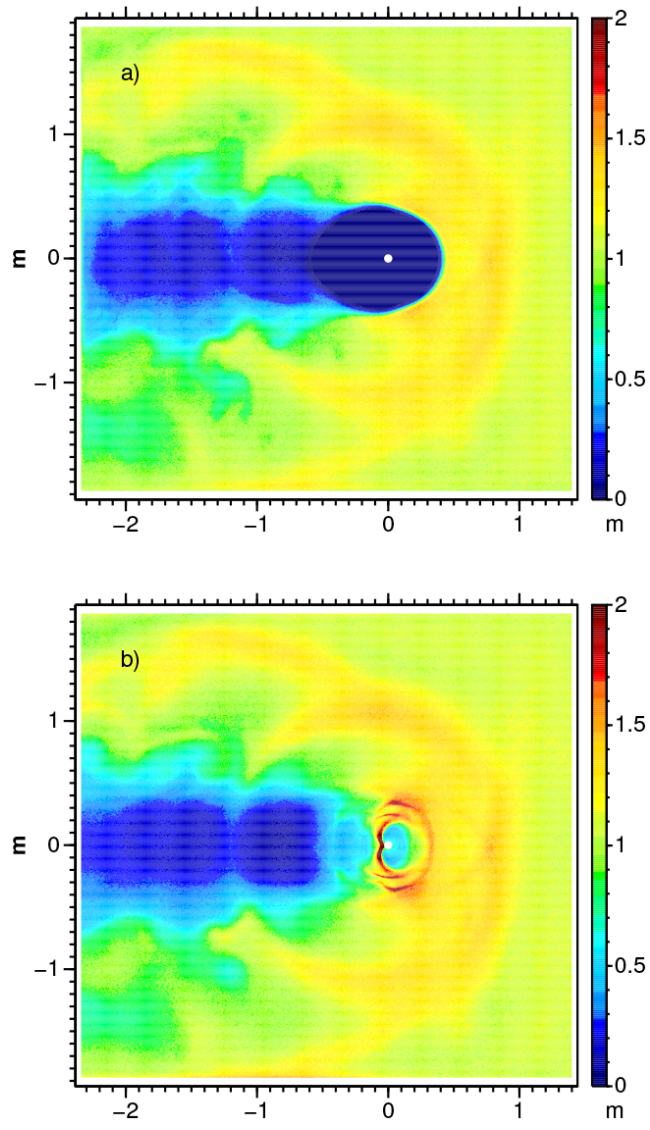

Figure 8. Electron density (a) and ion density (b) normalised to plasma-stream density in the final state $(t=2.43 \mathrm{~ms})$ of run Bz.

occurred, which had reduced the thrust. The smaller dynamic pressure of light-ion-bulk flow likely depresses the sheath less than in the oxygen case, and this effect is not included in Eq. (1), where the thrust is assumed to be linearly proportional to the ion mass. In the helium run (not shown), the behaviour is intermediate between the oxygen and proton runs. In Sect. 3.5 below, we will investigate the longer time behaviour of proton plasma, where a magnetic field is also included.

\subsection{Effect of electron temperature}

In all runs presented thus far, we have assumed that the electron temperature is $0.1 \mathrm{eV}$ (the same as ion temperature). In the $700-900 \mathrm{~km}$ altitude range, the electron temperature is actually often $0.2-0.3 \mathrm{eV}$. Figure 12 shows thrust behaviour of a run (named Te), which is otherwise identical to Baseline, but the electron temperature is set to $0.3 \mathrm{eV}$. The thrust remains essentially unchanged and the oscillation is damped. So, run Te suggests that a higher electron temperature improves stability.

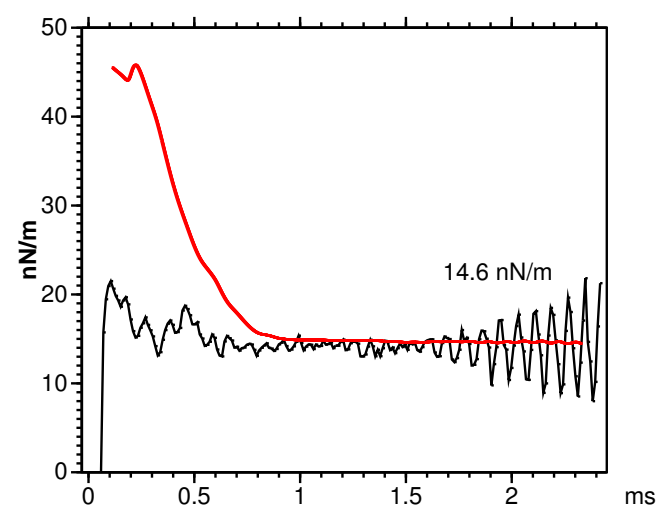

Figure 9. Run Helium: same as Fig. 2, but in helium plasma.

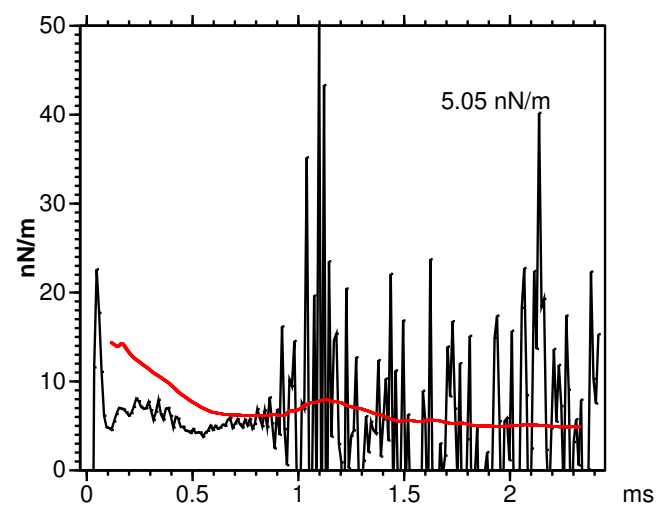

Figure 10. Run Proton: same as Fig. 2, but in proton plasma.

\subsection{Longer runs with magnetic field}

Figure 13 shows the longer time behaviour $\left(4 \times 10^{6}\right.$ time steps, $23.4 \mathrm{~ms}$ ) of the thrust in the presence of $X$-directed magnetic field. The result indicates that, after about $7 \mathrm{~ms}$, the thrust no longer decreases, while intermittent unstable wave activity continues. The asymptotic value of the thrust, as determined from the run, is $40.9 \mathrm{nN} \mathrm{m}^{-1}$, which is only $11 \%$ less than in Baseline when the difference in voltage between the runs $(311 \mathrm{~V}$ vs. $337 \mathrm{~V})$ is compensated for by a squareroot dependence. Hence, the simulation suggests that the instability caused by an $X$-directed magnetic field decreases the plasma-brake thrust only slightly at the baseline voltage.

Figure 14 shows the corresponding result for $Z$-directed magnetic field. The behaviour is similar, except that the fluctuation spectrum extends to somewhat lower frequencies. The final state thrust $38.7 \mathrm{nN} \mathrm{m}^{-1}$ is $17 \%$ lower than voltagecorrected Baseline.

Figure 15 shows the result with higher voltage $(757 \mathrm{~V}$, which is 2.4 times larger than Baseline) and $Z$-directed field. The fluctuations are strong and the thrust $53.8 \mathrm{nN} \mathrm{m}^{-1}$ is $27 \%$ lower than voltage-corrected Baseline. Thus, the relative gap between the $\mathrm{Bz}$ and $\boldsymbol{B}=0$ cases increases with voltage. The increase of the gap is slower than linear, however, 

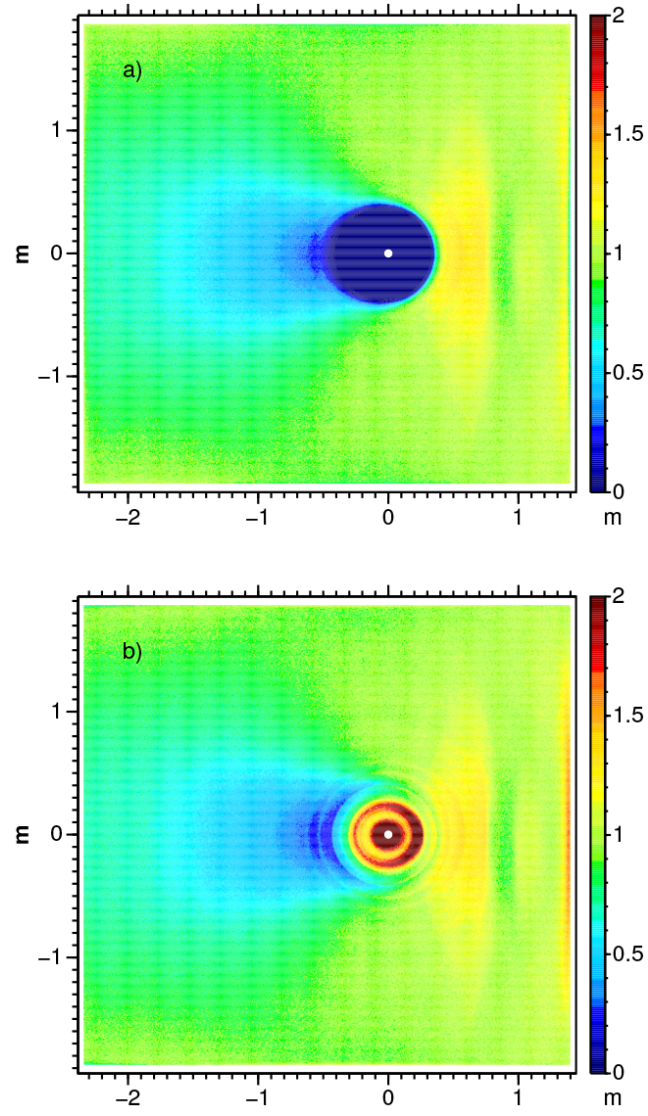

Figure 11. Electron density (a) and ion density (b) normalised to plasma-stream density in the final state $(t=2.43 \mathrm{~ms})$ of run Proton.

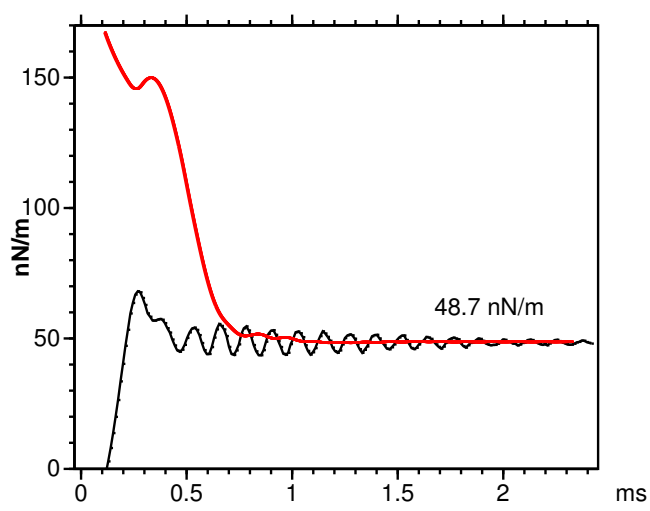

Figure 12. Run Te: same as Fig. 2, but with $T_{\mathrm{e}}=0.3 \mathrm{eV}$ instead of $0.1 \mathrm{eV}$.

since it increases from 17 to $\sim 27 \%$ if the voltage is made 2.4 times stronger. The thrust curve in Fig. 15 might not yet be completely stabilised at the end of the run, so the thrust estimate derived from the simulation, in this case, might be somewhat overestimated.

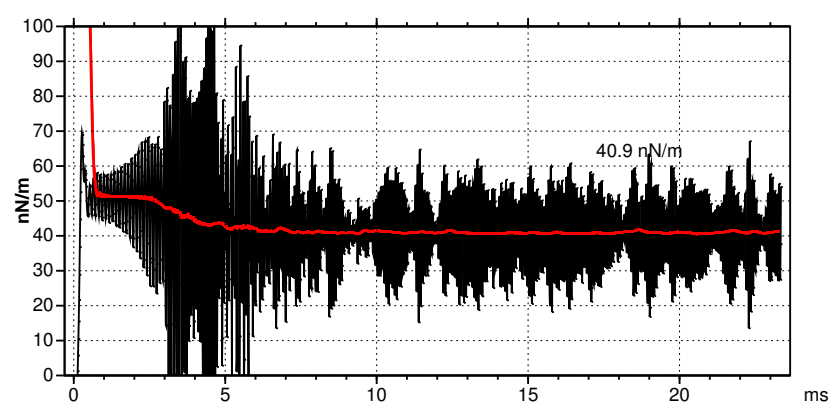

Figure 13. Run BxLong: $B_{x}=30000 \mathrm{nT}$ and $23.4 \mathrm{~ms}$ duration.

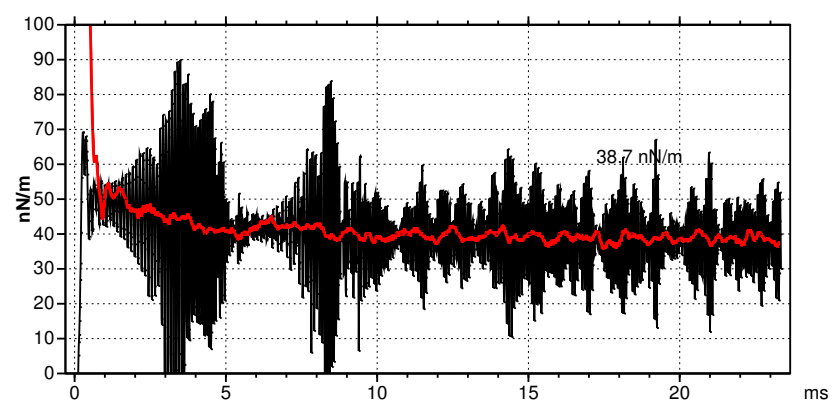

Figure 14. Run BzLong: $B_{z}=30000 \mathrm{nT}$ and $23.4 \mathrm{~ms}$ duration.

The $X$-directed magnetic-field case with high $856 \mathrm{~V}$ voltage is shown in Fig. 16. This run provides a positive surprise, since initial transients now die away quickly, after which the state is stable and the thrust is even somewhat larger than the Eq. (1) prediction. Thus, although the $X$-directed field at lower voltage is unstable and the instability lowers the thrust to a small extent (run BxLong), at higher voltage, the instability is absent and, instead of a small thrust reduction, we have a minor thrust enhancement.

Next, we look at the asymptotic state in the highly unstable proton case (see Sect. 3.3 above), where, in addition, a destabilising Bz magnetic field exists. The result is shown in Fig. 17. The fluctuations are strong, but the average magnitude of the thrust becomes stabilised during the run. Thus, even highly unstable behaviour does not cause a collapse of the ion sheath that surrounds the tether and whose spatial extent determines the thrust. Interestingly, the determined thrust of $2.91 \mathrm{nN} \mathrm{m}^{-1}$ at $V_{\mathrm{w}}=256 \mathrm{~V}$ is exactly (within three decimal places) equal to Eq. (1) prediction. The $\boldsymbol{B}=0$ value of the thrust, in case of proton plasma, would likely be slightly above Eq. (1) prediction, while Bz tends to slightly lower the thrust. The fact that the effects cancel out exactly may be fortuitous.

\subsection{Miscellaneous runs}

We also carried out a run in which the magnetic-field components had equal values: $B_{x}=B_{y}=B_{z}=30000 \mathrm{nT} / \sqrt{3}$. The result is an intermediate case of the $\mathrm{Bx}, \mathrm{By}$ and $\mathrm{Bz}$ 


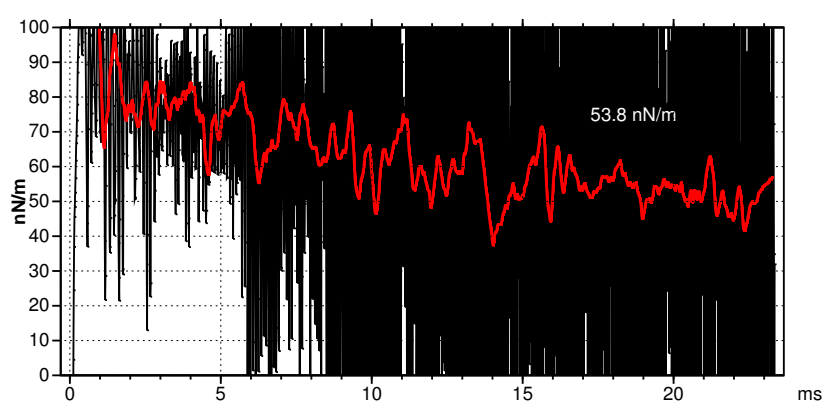

Figure 15. Run V1000BzLong: $V_{\mathrm{w}}=756.69 \mathrm{~V}, B_{z}=30000 \mathrm{nT}$ and $23.4 \mathrm{~ms}$ duration.

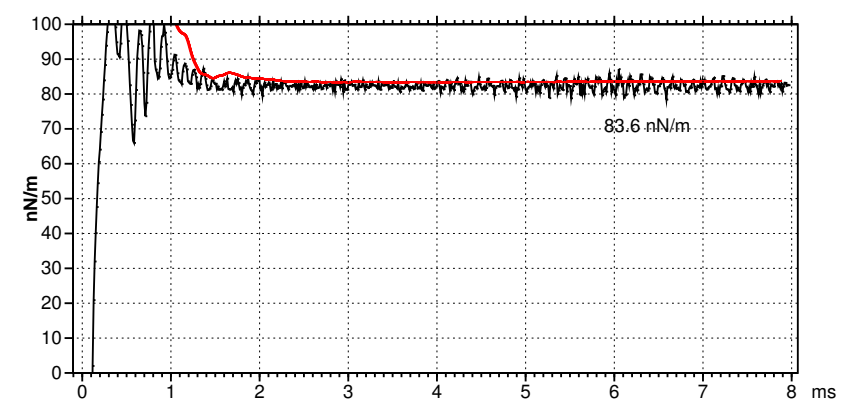

Figure 16. Run V1000Bx: $V_{\mathrm{W}}=856.07 \mathrm{~V}, B_{x}=30000 \mathrm{nT}$ and $8 \mathrm{~ms}$ duration.

runs: a very slow-growing oscillation appears. It therefore seems that the behaviour is stable if the magnetic field is predominantly in the $Y$ direction and otherwise unstable. The result suggests that, if the magnetic field has general orientation, the behaviour can be qualitatively interpolated from the $X$-, $Y$ - and $Z$-directed runs.

When the plasma density $n_{\mathrm{o}}$ is changed, all spatial scales in the electrostatic PIC simulation model scale naturally by the electron Debye length i.e. as $\sim 1 / \sqrt{n_{\mathrm{o}}}$. The only thing that breaks this scale invariance is the fixed value of the tether's effective electric radius $r_{\mathrm{w}}^{*}$. However, the effect of $r_{\mathrm{w}}^{*}$ is minor, because it only enters in Eq. (1) logarithmically. Thus, we can say that changing the plasma density $n_{\mathrm{o}}$ does not affect the stable/unstable nature of the solution or the thrust scales nearly as proportional to $\sqrt{n_{\mathrm{o}}}$. As a consistency check, we carried out one run to verify this behaviour.

We also performed a high-voltage run with a $Y$-directed field, V1000By (Table 2), with short $2.43 \mathrm{~ms}$ duration, because only a weak, stable oscillation is present and no instability is seen. As in the corresponding lower voltage run By, the determined thrust $80.7 \mathrm{nN} \mathrm{m}^{-1}$ is in close agreement with Eq. (1).

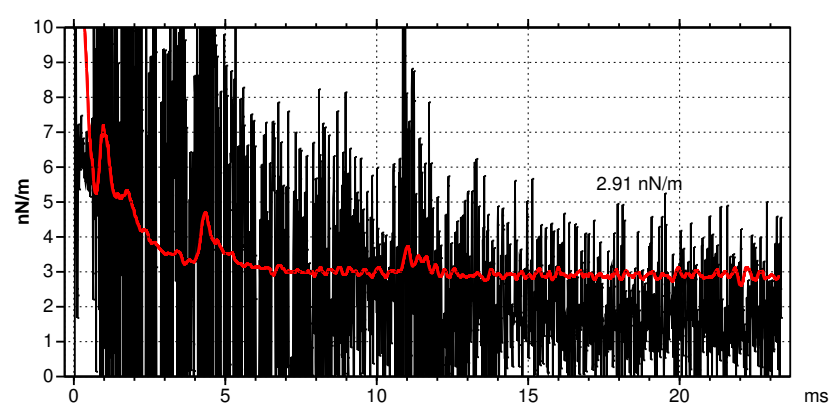

Figure 17. Run ProtonBzLong: proton plasma, $B_{z}=30000 \mathrm{nT}$ and 23.4 ms duration.

\section{Discussion}

In Eq. (1), the thrust is nearly linearly proportional to the ion mass and the simulations are in agreement with this at least qualitatively.

At $\sim 320 \mathrm{~V}$, the runs BxLong and BzLong suggest that, although the plasma sheath is unstable when an $X$ - or $Z$ dominant magnetic field is present, the thrust is reduced by the instability only modestly (11 and $17 \%$, respectively). When the voltage is increased to $\sim 800 \mathrm{~V}$, a $Z$-directed field is, again, unstable (V1000BzLong) and the thrust reduction is larger $(\sim 27 \%)$. In the case of an $X$-directed field, however, the instability is absent and there is actually a small thrust enhancement (V1000Bx). A $Y$-directed field is always stable and there is neither thrust reduction nor enhancement.

In other words, Eq. (1) is, in most cases, able to predict the thrust well. The only exception is that, if the magnetic field is aligned with the tether ( $Z$-directed case), then there is a moderate thrust reduction that increases with voltage. The relative reduction is $17 \%$ at $320 \mathrm{~V}$ and $\sim 27 \%$ at $760 \mathrm{~V}$ for $Z$-directed magnetic field.

When $\boldsymbol{B}=0$, the flow is stable with oxygen plasma and unstable with proton plasma (runs Baseline and Proton). When an instability sets in, the ion cloud, formed by ions passing near the tether, starts to oscillate at ion-plasma frequency. In the oxygen case the ion-plasma oscillation is four times slower than in the proton case. In the oxygen case, the flow has time to move by more than one ion-sheath diameter during one ion-plasma period, while, in the proton case, it moves only a fraction of the sheath diameter. Thus, in the proton-plasma case, if the sheath starts to oscillate, the oscillations have more opportunities to disturb the upstream flow and perhaps cause a positive non-linear feedback. Maybe this is why lighter ion-mass flow tends to be more unstable. Another way to arrive at a qualitatively similar conclusion is to note that the ion-bulk-flow kinetic energy $e V_{\mathrm{i}}=(1 / 2) m_{\mathrm{i}} v_{\mathrm{o}}^{2}$ is linearly proportional to the ion mass. Thus, the ratio $V_{\mathrm{w}} / V_{\mathrm{i}}$ is 16 times larger in proton-plasma flow than in $\mathrm{O}^{+}$ion flow. Any voltage-dependent instability should then occur at 16 times lower voltage in proton plasma than in $\mathrm{O}^{+}$plasma. 
Let us consider a vertical gravity-gradient-stabilised plasma-brake tether using the untilted dipole approximation for Earth's magnetic field. The untilted dipole approximation can be used in this case, because runs in which all field components had non-zero values seemed to interpolate smoothly from purely aligned runs (Sect. 3.6), i.e. exact orientation of the magnetic field does not seem to matter. In this approximation, in equatorial orbit, the magnetic field is $Y$-directed all the time and the thrust is consequently predicted well by Eq. (1). The same is true for polar orbit in low latitudes. Only for the high-latitude portion of a polar orbit is the thrust somewhat smaller than that which Eq. (1) predicts.

Let us look at a numeric example. At $1 \mathrm{kV}$ voltage, $3 \times$ $10^{10} \mathrm{~m}^{-3}$ density and oxygen plasma, the predicted plasmabrake thrust from Eq. (1) is $85 \mathrm{nN} \mathrm{m}^{-1}$. A $5 \mathrm{~km}$ long tether would produce $0.43 \mathrm{mN}$ braking force, which is equivalent to $13400 \mathrm{Ns}$ impulse per year. At $800 \mathrm{~km}$ altitude, reducing the orbital altitude by $100 \mathrm{~km}$ requires $52 \mathrm{~m} \mathrm{~s}^{-1}$ of delta $v$, thus the exemplary $5 \mathrm{~km}$ tether - mass $0.055 \mathrm{~kg}$, (Seppänen et al., 2013) - could lower the orbital altitude of a $260 \mathrm{~kg}$ object by $100 \mathrm{~km}$ over 1 year. The gathered oxygen-ion current per tether length is given by the orbital motion limited (OML) theory expression as

$$
\frac{\mathrm{d} I}{\mathrm{~d} z}=e n_{\mathrm{o}} \sqrt{\frac{2 e V_{\mathrm{w}}}{m_{\mathrm{i}}}} d_{\mathrm{w}}^{\mathrm{tot}}=8.4 \times 10^{-8} \mathrm{~A} / \mathrm{m},
$$

where $d_{\mathrm{w}}^{\text {tot }}$ is the total width of the four-wire tether $(160 \mu \mathrm{m})$. Hence, a $5 \mathrm{~km}$ tether gathers $0.4 \mathrm{~mA}$ current and consumes $0.4 \mathrm{~W}$ power in this plasma. In reality, the current is expected to be somewhat larger because of production of some secondary electrons from the tether when hit by oxygen ions. For comparison, the maximal Lorentz force produced by the tether (Cosmo and Lorenzini, 1997) (using the lengthaveraged current of $0.2 \mathrm{~mA}$ ) is only $0.03 \mathrm{mN}$, which is 14 times smaller than the plasma brake force in this case.

We did not test voltages higher than about $860 \mathrm{~V}$ in this paper. Going to higher voltages tends to increase the needed computing time, because the sheath becomes larger, so one needs more grid cells, particles and time steps to model its asymptotic evolution accurately. In a practical device, if one increases the negative voltage, at some point electron field emission from the surface of the tether wires starts to become an issue. Field emission adds to the ionic current gathered by the tether and hence increases power consumption. We think that the point at which field emission starts to become an issue is larger than $1 \mathrm{kV}$, but smaller than perhaps $3-5 \mathrm{kV}$. The value also depends on the geometry and possible coating of the tether. Before experimental knowledge about the plasma brake is obtained, it is not necessarily well motivated to try simulations with higher voltages than those presented in this paper.

\subsection{Correction of an earlier result}

The reference Janhunen (2010) contains an error: the quantities which are in the present paper denoted by $\tilde{V}$ and $V_{\mathrm{w}}$ were confused with each other. Therefore, the thrust vs. voltage relationship, as given in Janhunen (2010) was too optimistic. On the other hand, Janhunen (2010) used an earlier formula (Janhunen, 2009, Eq. 20) whose numerical coefficient had been found from a test particle calculation using solar-wind parameters. In the ionospheric plasma-brake case, the ratio between the tether voltage $V_{\mathrm{w}}$ and the bulk-flow energy $V_{\mathrm{i}}$ (in voltage units) is much larger than in the solar wind, and consequently ions are reflected backwards more efficiently in the ionosphere than in the solar wind, as is evident from the simulation results of the present paper. All in all, the results of Janhunen (2010) are not too far from reality: the error resulting from confusing $\tilde{V}$ and $V_{\mathrm{w}}$ and the inaccuracy resulting from using a formula suitable for solar wind roughly cancel each other out.

\section{Conclusions}

According to a series of electrostatic PIC simulations performed at different parameters, Eq. (1) can be used to predict plasma-brake thrust in ionospheric conditions. Only if the dominant component of the magnetic field is along the tether is the thrust reduced, and the relative reduction grows from 17 to $\sim 27 \%$ when the voltage increases from 320 to $760 \mathrm{~V}$. The thrust reduction is due to an instability that has ionic character.

The predicted performance of the plasma brake seems promising concerning satellite-deorbiting applications. For example, in $\mathrm{O}^{+}$plasma with $3 \times 10^{10} \mathrm{~m}^{-3}$ density and using $1 \mathrm{kV}$ voltage, a $5 \mathrm{~km}$ long plasma-brake tether weighing $0.055 \mathrm{~kg}$ could produce $0.43 \mathrm{mN}$ breaking force at altitude $\sim 800 \mathrm{~km}$, which is enough to reduce the orbital altitude of a $260 \mathrm{~kg}$ debris mass by $100 \mathrm{~km}$ over 1 year.

Acknowledgements. The work was partly supported by Academy of Finland, grant no. 250591.

Topical Editor K. Hosokawa thanks A. Sanchez-Torres and two anonymous referees for their help in evaluating this paper.

\section{References}

Birdsall, C. K. and Langdon, A. B.: Plasma physics via computer simulation, Adam Hilger, New York, 1991.

Cosmo, M. L. and Lorenzini, E. C.: Tethers in space handbook, NASA Marshall Space Flight Center, available at: http://ntrs.nasa.gov/archive/nasa/casi.ntrs.nasa.gov/ 19980018321_1998056794.pdf (last access: 26 September 2014), 1997.

Fog, A.: C++ vector class library, available at: http://www.agner. org/optimize/\#vectorclass (last access: 26 September 2014), 2013. 
Janhunen, P.: On the feasibility of a negative polarity electric sail, Ann. Geophys., 27, 1439-1447, doi:10.5194/angeo-27-14392009, 2009.

Janhunen, P.: Electrostatic plasma brake for deorbiting a satellite, J. Prop. Power, 26, 370-372, 2010.

Janhunen, P. and Sandroos, A.: Simulation study of solar wind push on a charged wire: basis of solar wind electric sail propulsion, Ann. Geophys., 25, 755-767, doi:10.5194/angeo-25-755-2007, 2007.

Janhunen, P., Toivanen, P. K., Polkko, J., Merikallio, S., Salminen, P., Haeggström, E., Seppänen, H., Kurppa, R., Ukkonen, J., Kiprich, S., Thornell, G., Kratz, H., Richter, L., Krömer, O., Rosta, R., Noorma, M., Envall, J., Lätt, S., Mengali, G., Quarta, A. A., Koivisto, H., Tarvainen, O., Kalvas, T., Kauppinen, J., Nuottajärvi, A., and Obraztsov A.: Electric solar wind sail: towards test missions, Rev. Sci. Instrum., 81, 111301, doi:10.1063/1.3514548, 2010.
Janhunen, P., Quarta, A. A., and Mengali, G.: Electric solar wind sail mass budget model, Geosci. Instrum. Method. Data Syst., 2, 85-95, doi:10.5194/gi-2-85-2013, 2013.

Sanmartin, J. R., Martinez-Sanchez, M., and Ahedo, E.: Bare wire anodes for electrodynamic tethers, J. Prop. Power, 9, 353-360, 1993.

Seppänen, H., Rauhala, T., Kiprich, S., Ukkonen, J., Simonsson, M., Kurppa, R., Janhunen, P., and Haeggström, E.: One kilometer $(1 \mathrm{~km})$ electric solar wind sail tether produced automatically, Rev. Sci. Instrum., 84, 095102, doi:10.1063/1.4819795, 2013. 